\title{
RERS2014 AND MRS2014: NEW HIGH-PRECISION RIGID EARTH ROTATION AND MOON ROTATION SERIES
}

\author{
V.V. Pashkevich \\ Central (Pulkovo) Astronomical Observatory of RAS \\ Pulkovskoe shosse, 65/1, 196140, St.Petersburg, Russia \\ e-mail: pashvladvit@yandex.ru
}

\begin{abstract}
Numerical investigation of the Earth and Moon rotational motion dynamics is carried out at a long time intervals. In our previous studies (Pashkevich, 2013), (Pashkevich and Eroshkin, 2011) the high-precision Rigid Earth Rotation Series (designated RERS2013) and Moon Rotation Series (designated MRS2011) were constructed. RERS2013 are dynamically adequate to the JPL DE422/LE422 (Folkner, 2011) ephemeris over 2000 and 6000 years and include about 4113 periodical terms (without attempt to estimate new subdiurnal and diurnal periodical terms). MRS2011 are dynamically adequate to the JPL DE406/LE406 (Standish, 1998) ephemeris over 418, 2000 and 6000 years and include about 1520 periodical terms. In present research have been improved the Rigid Earth Rotation Series RERS2013 and Moon Rotation Series MRS2011, and as a result have been constructed the new high-precision Rigid Earth Rotation Series RERS2014 and Moon Rotation Series MRS2014 dynamically adequate to the JPL DE422/LE422 ephemeris over 2000 and 6000 years, respectively. The elaboration of RERS2013 is carried out by means recalculation of sub-diurnal and diurnal periodical terms. The residuals in Euler angles between the numerical solution and RERS2014 do not surpass $3 \mu$ as over 2000 years. Improve the accuracy of the series MRS2011 is obtained by using the JPL DE422/LE422 ephemeris. The residuals in the perturbing terms of the physical librations between the numerical solution and MRS2014 do not surpass 8 arc seconds over 6000 years
\end{abstract}

Keywords: the rigid Earth rotation, the Moon rotation, ephemeris, iterative algorithm, leastsquares method, spectral analysis method.

\section{INTRODUCTION}

The main purposes of the present research are construction of the improved high-precision Rigid Earth Rotation Series RERS2014 (with including new sub-diurnal and diurnal periodical terms) and the high-precision Moon Rotation Series MRS2014 dynamically adequate to the JPL DE422/LE422 ephemeris, over 2000 and 6000 years, respectively. The numerical solution of the problem is obtained by solving the Lagrange differential equations of the second kind for the rigid Earth rotation with respect to the fixed ecliptic and equinox of epoch J2000 (Pashkevich, 2013). The orbital motions of the disturbing celestial bodies are defined by the DE422/LE422 ephemeris. The initial epoch of the numerical integration for the rigid Earth rotation problem is 1 January 2000 year $(\mathrm{JD}=2451545.0)$ and for the Moon rotation problem is 28 June 1969 year $(\mathrm{JD}=2440400.5)$. The mathematical model of the problem is described in detail in the papers (Eroshkin and Pashkevich, 1997), (Pashkevich 
and Eroshkin, 2011), (Eroshkin, Pashkevich and Brzeziński, 2002) and (Pashkevich, 2013). The discrepancies between the high-precision numerical solutions and the semi-analytical solutions for the rigid Earth and the Moon rotation problems with respect to the fixed ecliptic of epoch J2000 are investigated by the iterative algorithm, which used the least-squares method and the spectral analysis methods (Pashkevich and Eroshkin, 2005, 2010). Comparison of the new Series RERS2014 and MRS2014 with the previous solution RERS2013 (Pashkevich, 2013) and MRS2011 (Pashkevich and Eroshkin, 2011), respectively is carried out. The rigid Earth rotation problem is solved for the relativistic (kinematical) case in which the geodetic perturbations (the most essential relativistic perturbations) in the Earth rotation are taken into account. Investigation of the Moon rotation problem is carried out only for the Newtonian (dynamical) case. The problem of the Earth rotation is solved separately from the lunar rotation problem.

In our previous studies (Pashkevich, 2013), (Pashkevich and Eroshkin, 2011) the high-precision Rigid Earth Rotation Series (designated RERS2013) and Moon Rotation Series (designated MRS2011) were constructed. RERS2013 are dynamically adequate to the JPL DE422/LE422 (Folkner, 2011) ephemeris over 2000 and 6000 years and include about 4113 periodical terms (without attempt to estimate new sub-diurnal and diurnal periodical terms). The discrepancies in Euler angles between the numerical solution and RERS2013 do not surpass: 4 mas over 2000 years, 1 mas over 6000 years. MRS2011 are dynamically adequate to the JPL DE406/LE406 (Standish, 1998) ephemeris over 418, 2000 and 6000 years and include about 1520 periodical terms. The discrepancies between the numerical solution and MRS2011 do not surpass: 20 mas over 418 year time interval, 48 mas over 2000 year time interval, 8 arc seconds over 6000 year time interval.

The high-precision numerical integration method HIPPI (Eroshkin, 2000) was applied for the numerical solution of the rigid Earth rotation problem.

The high-precision numerical integration method (Belikov, 1990), with a number of modifications (Eroshkin et al., 1993), was applied for the numerical solution of the Moon rotation problem.

\section{ITERATIVE ALGORITHM OF THE PROBLEM}

The results of the numerical solutions of the problem are compared with the semi-analytical solutions of the studied body rotation (RERS2013 for the Earth or MRS2011 for the Moon). The residuals of these comparisons are studied by means the iterative algorithm:

1. Numerical solution of the studied body rotation (the rigid Earth or Moon) is implemented with the quadruple precision of calculations. The initial conditions are computed by the semi-analytical solution of the studied body rotation (RERS2013 for the Earth or MRS2011 for the Moon). Discrepancies between the numerical solution and the semi-analytical solution are obtained over all investigation time interval with 0.1 day spacing in Euler angles (1) for the Earth case or with 1 day spacing in the perturbing terms of the physical librations (2) for the Moon case. The expressions for these discrepancies are as follows

$$
\left.\begin{array}{l}
\Delta \psi=\sum_{k=0}^{8} \psi_{k} t^{k}+\sum_{j=1}^{4113} \sum_{k=0}^{4}\left[\psi_{S j k} \sin \left(v_{j 0}+v_{j 1} t\right)+\psi_{C j k} \cos \left(v_{j 0}+v_{j 1} t\right)\right] t^{k} \\
\Delta \theta=\sum_{k=0}^{8} \theta_{k} t^{k}+\sum_{j=1}^{4113} \sum_{k=0}^{4}\left[\theta_{S j k} \sin \left(v_{j 0}+v_{j 1} t\right)+\theta_{C j k} \cos \left(v_{j 0}+v_{j 1} t\right)\right] t^{k} \\
\Delta \varphi=\sum_{k=0}^{8} \varphi_{k} t^{k}+\sum_{j=1}^{4113} \sum_{k=0}^{4}\left[\varphi_{S j k} \sin \left(v_{j 0}+v_{j 1} t\right)+\varphi_{C j k} \cos \left(v_{j 0}+v_{j 1} t\right)\right] t^{k}
\end{array}\right\},
$$




$$
\left.\begin{array}{l}
\Delta \tau=\sum_{j=1}^{1520} \sum_{k=0}^{3}\left[\tau_{S j k} \sin \left(v_{j 0}+v_{j 1} t\right)+\tau_{C j k} \cos \left(v_{j 0}+v_{j 1} t\right)\right] t^{k} \\
\Delta \rho=\sum_{j=1}^{1520} \sum_{k=0}^{3}\left[\rho_{S j k} \sin \left(v_{j 0}+v_{j 1} t\right)+\rho_{C j k} \cos \left(v_{j 0}+v_{j 1} t\right)\right] t^{k} \\
\Delta I \sigma=I \sum_{j=1}^{1520} \sum_{k=0}^{3}\left[\sigma_{S j k} \sin \left(v_{j 0}+v_{j 1} t\right)+\sigma_{C j k} \cos \left(v_{j 0}+v_{j 1} t\right)\right] t^{k}
\end{array}\right\},
$$

where $\psi$ is the longitude of the ascending node of the Earth's dynamical equator on the fixed ecliptic J2000; $\theta$ is the angle of the inclination of the Earth's dynamical equator to the fixed ecliptic J2000; $\varphi$ is the proper rotation angle of the Earth between the ascending node of the Earth's dynamical equator and the principal axis of the minimum moment of inertia; $\tau, \rho$ and $\sigma$ are the perturbing terms of the physical librations of the Moon for the fixed ecliptic of epoch J2000 in the longitude, in the inclination and in the node longitude, respectively; $v_{j 0}, v_{j 1}$ are the phases and the frequencies of the corresponding semi-analytical solutions, respectively; $t$ is the time in the Julian days; $\psi_{k}, \theta_{k}, \varphi_{k}$ are the coefficients of the secular terms; $\psi_{s j k}, \theta_{s j k}, \varphi_{s j k}$, $\psi_{c j k}, \theta_{c j k}, \varphi_{c j k}, \tau_{s j k}, \rho_{s j k}, \sigma_{s j k}, \tau_{c j k}, \rho_{c j k}, \sigma_{c j k}$ are the coefficients of the periodic and Poisson terms.

2. Investigation of the discrepancies is carried out by the least squares method (LSQ) and by the spectral analysis (SA) method (described by Pashkevich and Eroshkin, (2005), (2010)). The sets of the frequencies of the semi-analytical solution are used without change. Only the coefficients of the systematic terms, the coefficients of the periodical terms and the coefficients of the Poisson terms are improved. The systematic, periodic and Poisson terms representing the new high-precision studied body rotation series (RERS2014_i for the Earth or MRS2014_i for the Moon (where $i$ is the number of iteration)) are determined:

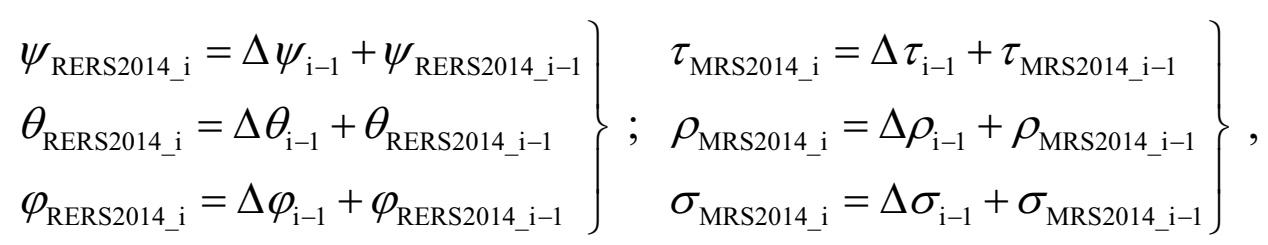

where $\quad \psi_{\text {RERS2014_0 }}=\psi_{\text {RERS2013 }}, \theta_{\text {RERS2014_0 }}=\theta_{\text {RERS2013 }}, \varphi_{\text {RERS2014_0 }}=\varphi_{\text {RERS2013 }}, \tau_{\text {MRS2014_0 }}=$ $\tau_{\mathrm{MRS} 2011}, \rho_{\mathrm{MRS2014} \_0}=\rho_{\mathrm{MRS} 2011}, \sigma_{\mathrm{MRS} 2014 \_0}=\sigma_{\mathrm{MRS} 2011}$.

3. Numerical solution of the studied body rotation is constructed anew with the new initial conditions, which are calculated by RERS2014_i (for the Earth case) or MRS2014_i (for the Moon case).

4. Steps 2 and 3 are repeated till the assumed convergence level of the discrepancies between the new numerical solution and the new semi-analytical solution (RERS2014_i for the Earth or MRS2014_i for the Moon) has been achieved.

\section{RESULTS}

The investigation of the rigid Earth rotation over 2000 years time interval is carried out with used DE422/LE422 ephemeris. The discrepancies between the numerical solutions and semianalytical series RERS2013 are depicted in Figure 1 blue colour. The convergence level was 
achieved after application of the second iteration of the iterative algorithm. So, the process of the iterative algorithm was finished at this step. As a result, the Rigid Earth Rotation Series RERS2014 was constructed, which include new recalculated the sub-diurnal and diurnal periodical terms and is dynamically adequate to the DE422/LE422 ephemeris over 2000 years. The discrepancies between the new numerical solutions and the semi-analytical solutions of RERS2014_2 do not surpass $3 \mu$ as over 2000 years for $\Delta \psi$ and $\Delta \varphi$ and do not surpass $1 \mu$ as over 2000 years for $\Delta \theta$ (presented in Figure 1 red colour).

\section{Mas}

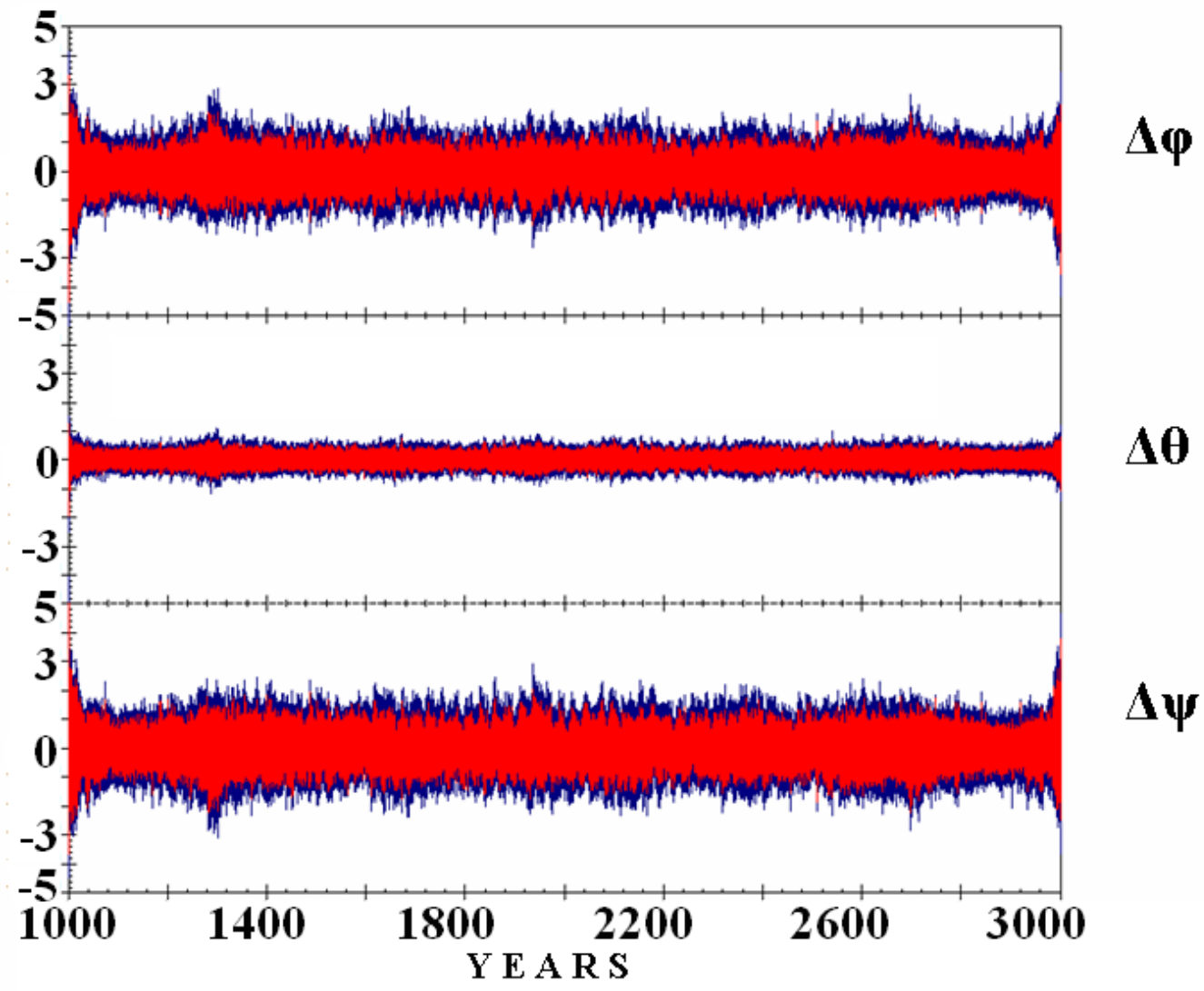

Fig. 1. Discrepancies between the numerical and RERS2013 solutions of the Earth rotation (blue colour) and between new numerical and RERS2014_2 solutions of the Earth rotation (red colour)

The investigation of the Moon rotation over 6000 years time interval is carried out with used DE422/LE422 ephemeris. The discrepancies between the numerical solutions and semianalytical series MRS2011 (dynamically adequate to the DE406/LE406 ephemeris) are depicted in Figure 2 blue colour. The convergence level was achieved after application of the first iteration of the iterative algorithm. So, the process of the iterative algorithm was finished at this step. As a result, the Moon Rotation Series MRS2014 was constructed, which is dynamically adequate to the DE422/LE422 ephemeris over 6000 years. The discrepancies between the new numerical solutions and the semi-analytical solutions of MRS2014_1 do not surpass 8 arc seconds over 6000 years for $\Delta \mathrm{I} \sigma$ do not surpass 4 arc seconds over $6 \overline{000}$ years for $\Delta \rho$ and do not surpass 0.6 arc seconds over 6000 years for $\Delta \tau$ (presented in Figure 2 red colour). 


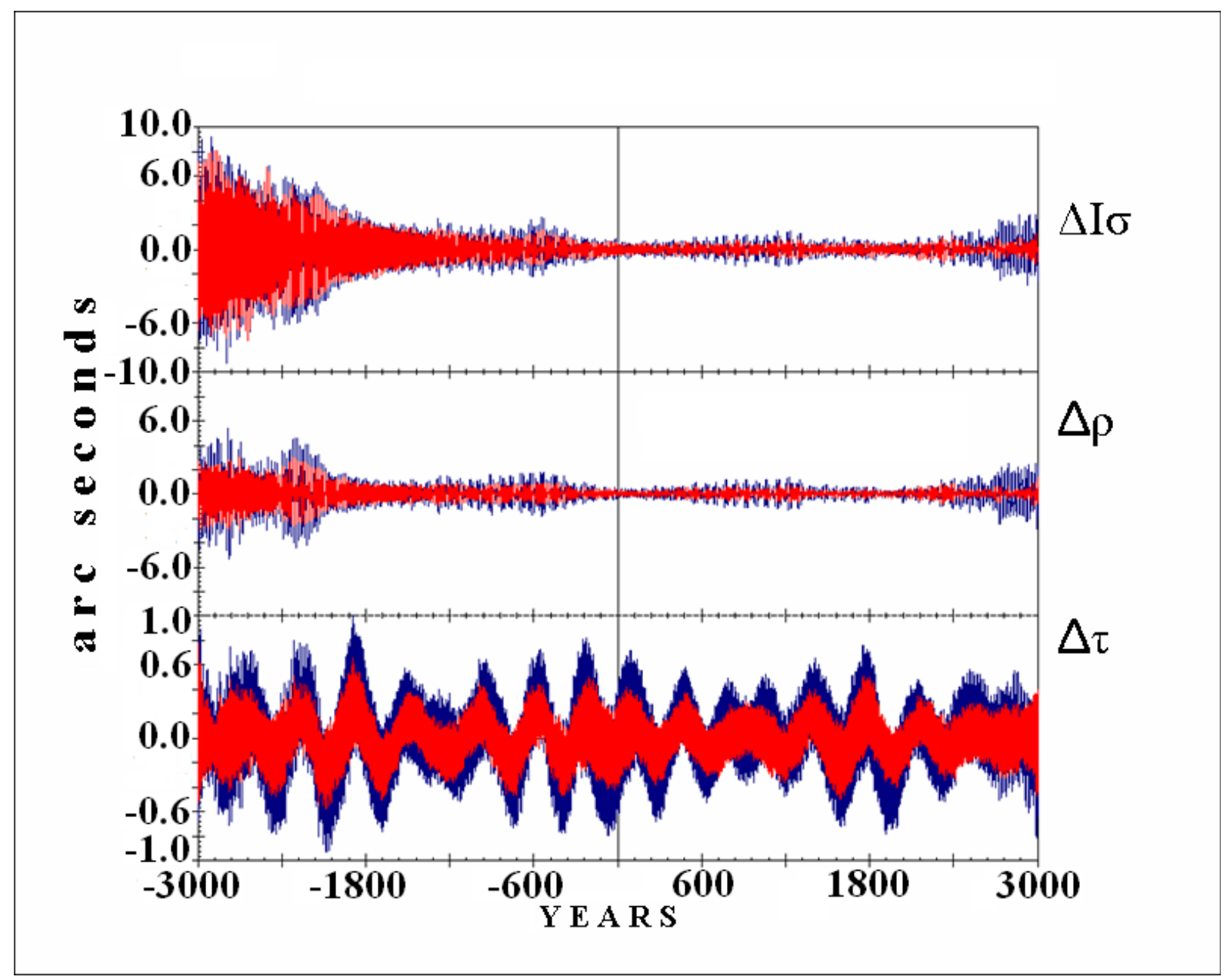

Fig. 2. Discrepancies between the numerical and MRS2011 solutions of the Moon rotation (blue colour) and between new numerical and MRS2014_1 solutions of the Moon rotation (red colour)

Thus, the result of the comparison on 2000 years for the Earth case and 6000 years for the Moon case demonstrates a good consistency of new RERS2014 and MRS2014 series, respectively, with the DE422/LE422 ephemeris.

\section{CONCLUSIONS}

The new improved high-precision Rigid Earth Rotation Series RERS2014 (with including new sub-diurnal and diurnal periodical terms) dynamically adequate to the DE422/LE422 ephemeris over 2000 years have been constructed. RERS2014 include about 4113 periodical terms. The residuals between the numerical solution and RERS2014 do not surpass $3 \mu$ as over 2000 years.

The new high-precision Moon Rotation Series MRS2014 dynamically adequate to the DE422/LE422 ephemeris over 6000 years has been constructed. MRS2014 include about 1520 periodical terms. The residuals between the numerical solution and MRS2014 do not surpass 8 arc seconds over 6000 years. It means a good consistency of the MRS2014 series with the DE422/LE422 ephemeris.

It should be noted the residuals between the numerical solution and RERS2014 are far less than the residuals between the numerical solution and MRS2014. This fact is partly due to the different time intervals of comparison residuals in these investigations. The time interval of comparison residuals for the Moon is thrice as much than for the Earth. But main explanation 
is largely due to the various level of the accuracy of the solutions for each studied body rotation (the rigid Earth or Moon). At the present time the definition of the force function for the Earth gravitational interaction with disturbing celestial bodies is a lot better than the force function for the Moon gravitational interaction with disturbing celestial bodies.

Acknowledgements. The investigation was carried out at the Central (Pulkovo) Astronomical Observatory of the Russian Academy of Science and the Space Research Centre of the Polish Academy of Science, under a financial support of the Cooperation between the Polish and Russian Academies of Sciences, Theme No 34 and the Polish national science foundation NCN under grant No. DEC-2012/05/B/ST10/02132.

\section{REFERENCES}

Eroshkin G.I., Pashkevich V.V. (1997) Numerical Simulation of the Rotational Motion of the Earth and Moon, Dynamics and Astrometry of Natural and Artificial Celestial Bodies. IAU Colloquium 165 (I.M.Wytrzyszczak, J.H.Lieske, R.A.Feldman, eds), Kluwer, Dordrecht, pp. 275-280.

Standish E.M. (1998) JPL Planetary and Lunar Ephemerides, DE405/LE405, JPL IOM 312.F-98-048.

Eroshkin G.I.: (2000) High-Precision method of the Numerical Solution of the Celestial Mechanic problems based on Chebishev Polynomial Interpolation, Proc. Astrometry, Geodynamics and Celestial Mechanics at the turn of XXIth century, (The Conference of the St.Petersburg in the IPA RAS, 19-23 June 2000), pp. 229-230 (in Russian).

Eroshkin G.I., Pashkevich V.V. and Brzezińnski A., (2002) Extension of the high-precision numerical theory of the rigid Earth rotation to the case of a long time interval, Artificial Satellites. Vol. 37., No. 4, pp. 169-183.

Pashkevich V.V., Eroshkin G.I. (2005) Choice of the optimal spectral analysis scheme for the investigation of the Earth rotation problem, Proc. Journées Systèmes de Référence Spatio-Temporels 2005, eds. Brzeziński A., Capitaine N., Kolaczek B.,Warsaw 2005, 105-109.

Pashkevich V.V., Eroshkin G.I. (2010) Application of the spectral analysis for modeling the rotations of the Moon, Artificial Satellites, Vol. 45. No. 4. (DOI: 10.2478/v10018-0110004-4), pp. 153-162.

Folkner W.F. (2011) JPL Planetary and Lunar Ephemerides : Export Information http://iaucomm4.jpl.nasa.gov/README.html

Pashkevich V.V., Eroshkin G.I. (2011) Construction of the numerical and semi-analytical solutions of the Moon rotation, Proc. Journées Systèmes de Référence Spatio-Temporels 2011, ed. N.Capitaine, Vienna, 19-21 September 2011, pp. 205-208.

Pashkevich V.V. (2013) Construction of the numerical and semi-analytical solutions of the rigid Earth rotation at a long time intervals, Journal of Planetary Geodesy, Artificial Satellites, Vol. 48, No. 1, (DOI: 10.2478/arsa-2013-0003), 25-37.

Received: 2014-12-15,

Reviewed: 2015-01-20, by V. Zharov, Accepted: 2015-02-27. 